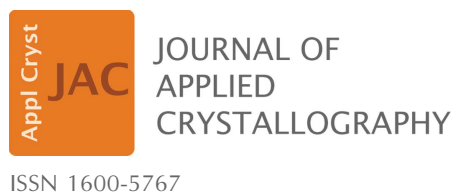

ISSN 1600-5767

Received 6 February 2017

Accepted 17 May 2017

Edited by J. M. García-Ruiz, Instituto Andaluz de Ciencias de la Tierra, Granada, Spain

Keywords: protein X-ray crystallography; aperiodic crystals; incommensurate modulation; superspace groups.

Supporting information: this article has supporting information at journals.iucr.org/j

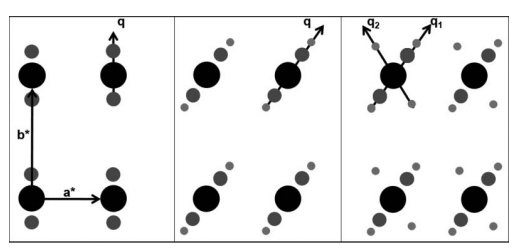

OPEN $\odot$ ACCESS

\section{How to assign a $(3+1)$-dimensional superspace group to an incommensurately modulated biological macromolecular crystal}

\author{
Jason Porta, ${ }^{\mathrm{a}, \mathrm{b}}$ Jeff Lovelace $^{\mathrm{a}}$ and Gloria E. O. Borgstahl ${ }^{\mathrm{a}, \mathrm{b} *}$
}

a'The Eppley Institute for Research in Cancer and Allied Diseases, University of Nebraska Medical Center, 986805 Nebraska Medical Center, Omaha, NE 68198, USA, and ${ }^{\mathbf{b}}$ Department of Biochemistry and Molecular Biology, University of Nebraska Medical Center, 985870 Nebraska Medical Center, Omaha, NE 68198, USA. *Correspondence e-mail: gborgstahl@unmc.edu

Periodic crystal diffraction is described using a three-dimensional (3D) unit cell and 3D space-group symmetry. Incommensurately modulated crystals are a subset of aperiodic crystals that need four to six dimensions to describe the observed diffraction pattern, and they have characteristic satellite reflections that are offset from the main reflections. These satellites have a non-integral relationship to the primary lattice and require $\mathbf{q}$ vectors for processing. Incommensurately modulated biological macromolecular crystals have been frequently observed but so far have not been solved. The authors of this article have been spearheading an initiative to determine this type of crystal structure. The first step toward structure solution is to collect the diffraction data making sure that the satellite reflections are well separated from the main reflections. Once collected they can be integrated and then scaled with appropriate software. Then the assignment of the superspace group is needed. The most common form of modulation is in only one extra direction and can be described with a $(3+1) D$ superspace group. The $(3+1) D$ superspace groups for chemical crystallographers are fully described in Volume $\mathrm{C}$ of International Tables for Crystallography. This text includes all types of crystallographic symmetry elements found in small-molecule crystals and can be difficult for structural biologists to understand and apply to their crystals. This article provides an explanation for structural biologists that includes only the subset of biological symmetry elements and demonstrates the application to a real-life example of an incommensurately modulated protein crystal.

\section{Introduction}

Our laboratory is interested in solving the structures of incommensurately modulated protein crystals. These crystals have a fascinating diffraction pattern with satellite reflections surrounding the main reflections. Commensurate and incommensurate macromolecular crystallography, with examples of such effects, as well as twinning and multiple crystal cases were reviewed by Helliwell (2008) and are also discussed in Chapter 8 of Rupp's Biomolecular Crystallography text book (Rupp, 2010). This paper concerns our symmetry analysis of the diffraction from $(3+1)$-dimensionally incommensurately modulated crystals of profilin:actin (PA) (Lovelace et al., 2008; Porta et al., 2011). This publication relies heavily on our study of an article by van Smaalen (2005), Chapters 1, 2 and 3 of van Smaalen's textbook on Incommensurate Crystallography (van Smaalen, 2007) and International Tables for Crystallography, Volume C, Chapter 9.8, Incommensurate and Commensurate Modulated Structures, by Janssen et al. (1999). We also studied Schönleber's lectures on Introduction to Superspace Symmetry 
from the Workshop on Structural Analysis of Aperiodic Crystals held in Bayreuth, Germany, and an article by Wagner \& Schönleber (2009). Although these are excellent sources, they were written for small-molecule crystallographers and physicists and use language and examples that are not encountered in macromolecular crystallography. Therefore, we decided to write this paper for the next biological crystallographer who chooses to solve a modulated crystal, so that it will not be so difficult for them to understand and to confidently assign their superspace group to the crystal diffraction data.

In this article the nomenclature common to periodic threedimensional (3D) crystals is used with adaptations to a fourth dimension as needed (Janssen et al., 1999). It is noteworthy that in much of the aperiodic literature another formalism is used, where subscripts $i=1,2,3$ are used to indicate the space directions (van Smaalen, 2007). This makes it easier to add more dimensions as needed. Hence, the symbols $(\mathbf{a}, \mathbf{b}, \mathbf{c}),(x, y$, $z),(h k l)$ and $(\alpha, \beta, \gamma)$ used in this publication correspond to $\left(\mathbf{a}_{1}, \mathbf{a}_{2}, \mathbf{a}_{3}\right),\left(x_{1}, x_{2}, x_{3}\right),\left(h_{1} h_{2} h_{3}\right)$ and $\left(\sigma_{1}, \sigma_{2}, \sigma_{3}\right)$, respectively, in aperiodic crystallography. Vectors are in bold and scalar coefficients are in italics. This is pointed out here to help avoid confusion when reading the aperiodic literature.

Crystal periodicities can be categorized into three types (Fig. 1). The first is the most common case, where the crystal is periodic and the unit-cell contents are duplicated closely by the lattice translations (Fig. 1a). The second type is the case of a commensurate modulation. Here, the spacing of the satellite

\section{Periodic}

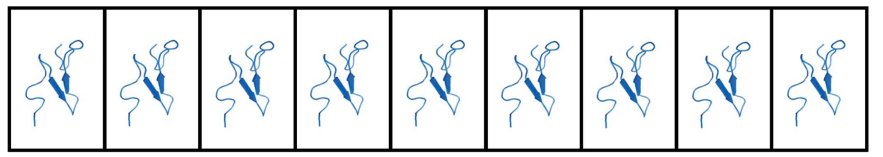

(a)

\section{Commensurate $\left(q=0.25 b^{*}\right)$}

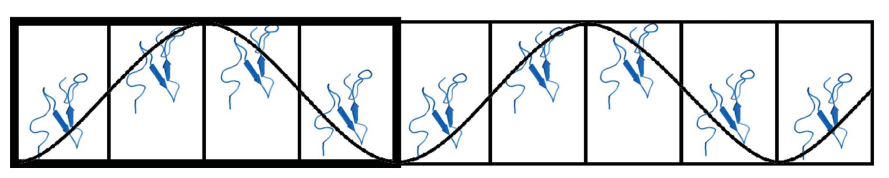

(b)

Incommensurate $\left(q=0.2853 \ldots b^{*}\right)$

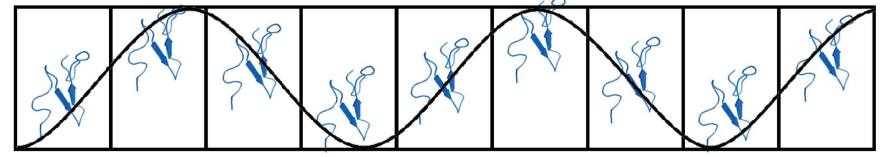

(c)

Figure 1

Three categories of crystals. (a) Periodic case with identical unit cells. (b) Commensurate modulation. (c) Incommensurate modulation with harmonic modulation wave. Protein structure from PDB entry 2rro. This figure is reproduced from Porta et al. (2011). The modulation here is kept simple for the purposes of instruction. Modulations can be complex and involve translations, rotations, variations in occupancies, subdomains and/ or a combination. reflections relative to the main reflections is a rational value. The diffraction pattern can be indexed and integrated with any protein crystallography data reduction software with three integer indices as a supercell. In Fig. 1(b), the $\mathbf{q}$ vector which is used in aperiodic crystallography to index the satellite reflections relative to the main reflection has a rational value of 0.25 (or 1/4) and the modulation of the structure repeats every four unit cells. This means that the lattice parameters for indexing the satellite reflections are integer multiples ( 1 , $2, \ldots, n)$ and the crystal structure can be described with a supercell (Wagner \& Schönleber, 2009). The third type is the case of an incommensurately modulated crystal. Here, at least one component of the $\mathbf{q}$ vector is irrational and cannot be calculated with a simple fraction (Fig. 1c). An accurate description of an incommensurately modulated crystal can only be obtained by describing the diffraction pattern with $\mathbf{q}$ vectors.

When an incommensurately modulated diffraction pattern is observed in protein crystallography, the sample is typically discarded in favour of a better behaving sample that can be processed with standard macromolecular crystallography software. As a consequence, incommensurately modulated macromolecular crystals are rarely reported and these types of structural modulations in the context of a macromolecular crystal are poorly understood. PA crystals can be chemically induced to form a peculiar incommensurately modulated diffraction pattern. More than 28 years ago (Schutt et al.,1989) it was found that when PA crystals are driven to a phase transition boundary by exposing the crystals to conditions known to promote actin filament formation they transform into an incommensurately modulated state that is thought to contain a superstructure of structural intermediates. By varying the solution conditions PA can be crystallized in either an 'open' or a 'closed/tight' state that corresponds to the nucleotide binding site opening and closing (Chik et al., 1996; Porta, 2011; Schutt et al., 1993). These two states are accompanied by a change in the $c$ unit-cell dimension from 186 to

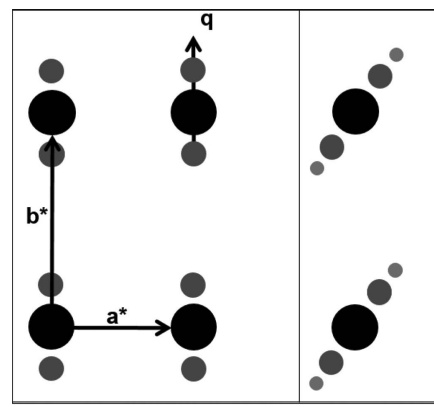

(a)

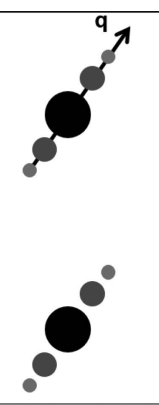

(b)

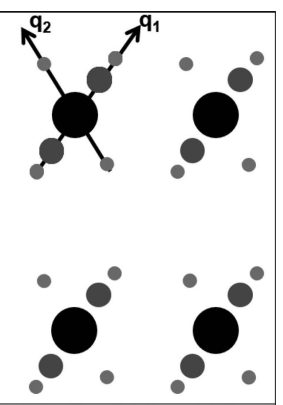

(c)
Figure 2

$(3+d) \mathrm{D}$ incommensurate diffraction scenarios, where $d=1$, 2, or 3. (a) $(3+1) D$ modulation with a single $\mathbf{q}$ vector parallel to $\mathbf{b}^{*}$ and first-order satellites $(m= \pm 1)$. $(b)(3+1) \mathrm{D}$ with second-order satellites $(m= \pm 2)$ and a single $\mathbf{q}$ vector in the $a b$ plane. $(c)(3+2) \mathrm{D}$ diffraction with two $\mathbf{q}$ vectors. The large black circles represent the 'main' reflections and the smaller grey and light-grey circles represent the 'satellite' reflections. This figure was reproduced from Porta et al. (2011). 
Table 1

Eval15 data processing statistics for incommensurately modulated PA diffraction data.

Data for the highest-resolution shell are in parentheses.

\begin{tabular}{|c|c|c|c|}
\hline Crystal name & xMod1 & xMod2 & gMod3 \\
\hline Crystal treatment $\dagger$ & $\begin{array}{l}\text { Crosslinked } 25 \% \text { glutaraldehyde in } \\
0.001 \mathrm{~N} \mathrm{HCl}, 7 \mathrm{M} \text { sodium formate }\end{array}$ & $\begin{array}{l}\text { Crosslinked } 25 \% \text { glutaraldehyde in } \\
0.001 \mathrm{~N} \mathrm{HCl}, 7 \mathrm{M} \text { sodium formate }\end{array}$ & Soaked in $70 \%(w / v)$ D-glucose \\
\hline Beamline & Rigaku FRE/VariMaxHR & NSLS X4C & Rigaku FRE/VariMaxHR \\
\hline Maximum resolution $(\AA)$ & 3.0 & 2.5 & 2.4 \\
\hline Completeness $(\%)$ & $98.2(85.9)$ & $96.3(95.5)$ & $99.9(100)$ \\
\hline \multicolumn{4}{|l|}{ Unit-cell parameters } \\
\hline$a(\AA)$ & 37.3 & 37.6 & 38.0 \\
\hline$\alpha, \beta, \gamma\left({ }^{\circ}\right)$ & 90 & 90 & 90 \\
\hline q vector, order & $0 \mathbf{a}^{*}+0.2829 \mathbf{b}^{*}+0 \mathbf{c}^{*}, d=1$ & $0 \mathbf{a}^{*}+0.2628 \mathbf{b}^{*}+0 \mathbf{c}^{*}, d=1$ & $0 \mathbf{a}^{*}+0.2700 \mathbf{b}^{*}+0 \mathbf{c}^{*}, d=1$ \\
\hline$\langle I / \sigma\rangle[$ main; satellites] & {$[15.2(4.2) ; 2.2(1.4)]$} & {$[3.4(1.6) ; 1.9(0.73)]$} & {$[7.5(2.2) ; 7.6(1.8)]$} \\
\hline$\langle I / \sigma\rangle[$ main; satellites] 23-4.0 & {$[15.9(10.8) ; 3.1(2.2)]$} & {$[4.8(4.8) ; 2.9(3.1)]$} & {$[14.6(14.5) ; 15.8(15.9)]$} \\
\hline No. unique reflections [main; satellites] & {$[10669 ; 21532]$} & [20 541; 39 852] & {$[23631 ; 42530]$} \\
\hline Laue group & 222 & 222 & 222 \\
\hline$R_{\mathrm{sum}}(\%) \ddagger$ & $8.2(56.3)$ & $11.4(33.4)$ & $10.6(37.3)$ \\
\hline
\end{tabular}

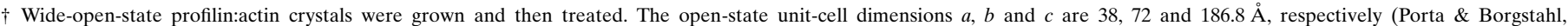
2012). $\neq R_{\text {sym }}=\sum_{h k l} w_{i}\left(\left|I_{i}-I_{\text {mean }}\right|\right) / \sum_{h k l}\left(I_{i}\right)$.

$172 \AA$, respectively. Incommensurate diffraction was obtained using precession photography at room temperature from either open or closed states by shifting the $\mathrm{pH}$ to 6.0 , a condition known to cause profilin to diffuse away from actin and actin filaments to form in vitro (Carlsson, 1979; Oda et al., 2001; Chik, 1996). This research provided the foundation for our continued studies.

Incommensurate modulations within crystals are a result of a displacement modulation that forms but does not align with the spacing of the basic unit cell. In the resulting diffraction pattern satellite reflections appear near the normal main reflections (see Fig. 2). In the periodic state, all reflections can be indexed by the three integer indices $h, k$ and $l$ such that

$$
\mathbf{H}=h \mathbf{a}^{*}+k \mathbf{b}^{*}+l \mathbf{c}^{*},
$$

where $\mathbf{a}^{*}, \mathbf{b}^{*}$ and $\mathbf{c}^{*}$ are the reciprocal lattice vectors of the main reflections and basic unit cell. With satellite reflections, the diffraction pattern becomes $(3+d)$ dimensional, where $d$ is the number of satellite directions. The most common form of modulation is in only one extra direction $(d=1)$, and the diffraction patterns for these crystals have satellite reflections on either side of the main reflection (see Figs. $2 a$ and $2 b$ ). This is called a $(3+1) \mathrm{D}$ modulated crystal. The diffraction pattern for this case can be indexed by the introduction of a single $\mathbf{q}$ vector such that

$$
\mathbf{H}=h \mathbf{a}^{*}+k \mathbf{b}^{*}+l \mathbf{c}^{*}+m \mathbf{q} .
$$

The positions of the satellite reflections are given by the $\mathbf{q}$ vector

$$
\mathbf{q}=\alpha \mathbf{a}^{*}+\beta \mathbf{b}^{*}+\gamma \mathbf{c}^{*}
$$

A modulation wave can be parallel to one of the reciprocal lattice vectors, and in this case two of the scalar $\mathbf{q}$ coefficients in (3) would be zero. In more complicated cases two or three of the $\mathbf{q}$ coefficients can be nonzero (Fig. $2 b$ ). Also, multipleorder satellites evenly spaced from the main reflections can exist (see Figs. $2 b$ and $2 c$ ). This is represented by the integer value $m$ in equation (2). Interestingly satellites and multiorder satellites are predominantly in the high-resolution bins of data (see Fig. 5 of Lovelace et al., 2010). In 2008, the Borgstahl laboratory was able to reproduced the incommensurately modulated PA crystals from the Schutt laboratory and measured a single-rotation-style diffraction image from a room-temperature protein crystal (Lovelace et al., 2008). The data were indexed and the first $\mathbf{q}$ vector was measured for a macromolecular crystal. Research progress was hindered by the reversibility of the modulation at room temperature, perhaps due to crystal heating or radiation damage from the SuperBright FRE X-ray generator, which prevented the collection of a full set of diffraction data. We have since learned to cryocool crystals that were first crosslinked with acidic glutaraldehyde at room temperature and then cryopreserved with sodium formate (named xMod1 and xMod2, Table 1) and more recently not crosslinked and cryopreserved with D-glucose $(\mathrm{gMod} 3$, Table 1$)$.

All of the $(3+d) \mathrm{D}$ superspace groups have been tabulated for $d=1,2$ or 3 . For $d=1$ there are 775 groups, for $d=2$ there are 3338 groups and for $d=3$ there are 12584 groups (Stokes et al., 2011). A web site has been developed for searching all $775(3+1)$ D superspace groups listed in International Tables for Crystallography (http://it.iucr.org/resources/finder/; Orlov et al., 2008). These numbers are greatly reduced for biological crystals as there are only 65 chiral (or biological) threedimensional space groups. Then there are only $135(3+1) \mathrm{D}$, $368(3+2) \mathrm{D}$ and $1019(3+3) \mathrm{D}$ chiral superspace groups (van Smaalen et al., 2013). An excellent primer to the threedimensional space groups was written by Dauter \& Jaskolski (2010) and can be used to review the symmetry elements 
found in protein crystals (Dauter \& Jaskolski, 2010). Modulated PA crystals have a $(3+1)$ D-type superspace group because they are modulated in only one direction.

The three cryocooled modulated PA data sets (Table 1) all have basic three-dimensional unit cells like that of the PA

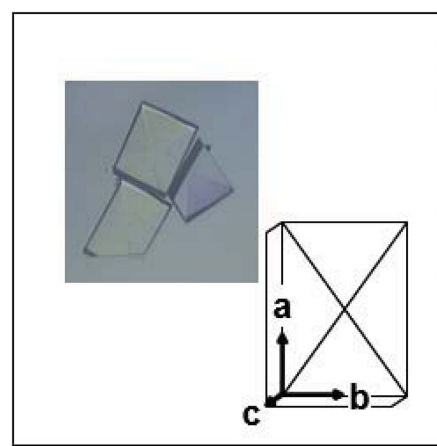

(a)

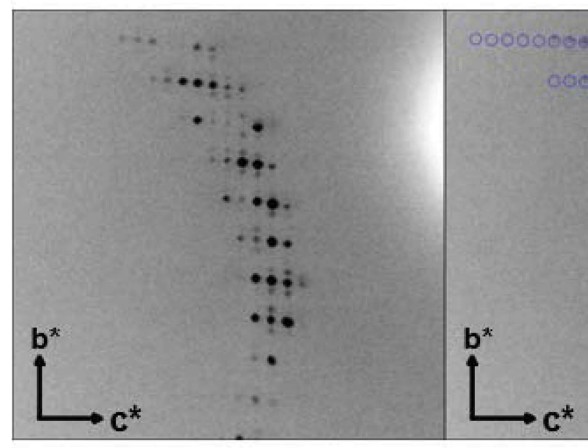

(c)

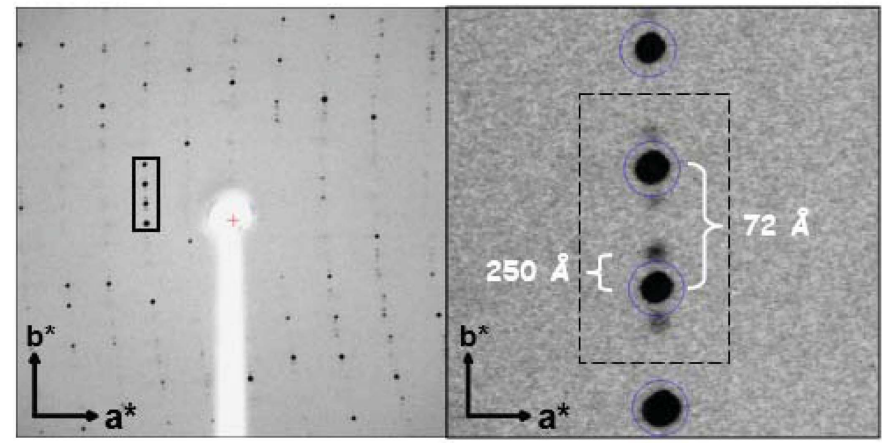

(e)

$(f)$

Figure 3

Profilin:actin crystals and diffraction patterns. (a) Typical profilin:actin crystal with a diagonal cross along the 001 plane. (b) Normal periodic diffraction pattern (Porta \& Borgstahl, 2012). (c) Incommensurately modulated diffraction pattern from crystal gMod3 with satellite reflections along $\mathbf{b}^{*}$. (d) Same as part (c) but with the main reflections circled in blue from the predicted positions calculated by the CrystalClear software (Rigaku, Tokyo, Japan). Note the satellite reflections are not predicted; only the main reflections are predicted. (e) Incommensurately modulated diffraction pattern from crystal xMod1 with satellite reflections in the $\mathbf{b}^{*}$ direction. $(f)$ Close-up of the modulated diffraction with approximated unit-cell dimensions. The blue circles are predicted reflection positions. Again, the satellite reflections are not predicted; only the main reflections are predicted. Images were collected at a distance of $300 \mathrm{~mm}$ with $15 \mathrm{~min}$ exposures and an oscillation of $\Delta \varphi=0.5^{\circ}$. Parts $(a)$ $(c),(e)$ and $(f)$ of this figure were reproduced from Lovelace et al. (2008). Note that the crystal diffraction data were processed with Eval15, not the CrystalClear software. CrystalClear was used here for the purposes of illustration only. open-state crystals and have satellite reflections along $\mathbf{b}^{*}$ (Fig. 3). The crystals differ in their resolution of diffraction and the extent of modulation, as indicated by their $\mathbf{q}$ vector and satellite intensity strength. The $q$ spacing of the satellite from the main reflections varies from 0.2628 to 0.2829 . A demonstration of their similarity to and differences from each other and from open-state crystals was made by calculating $R_{\text {merge }}$ between data sets (Table 2). The easiest $R_{\text {merge }}$ to calculate employs the main reflections only. This statistic demonstrates that the crystals are not isomorphous with each other. When the satellite intensities are added to the main
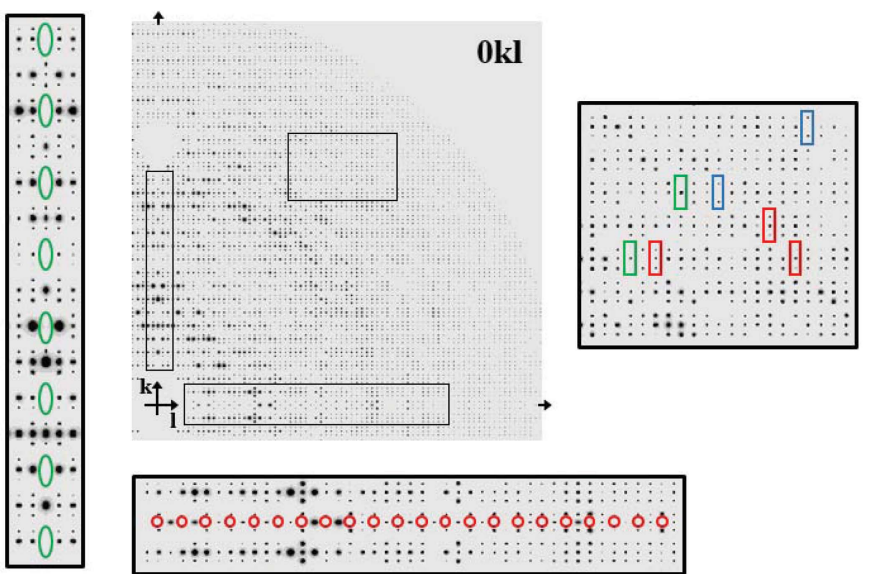

(a)

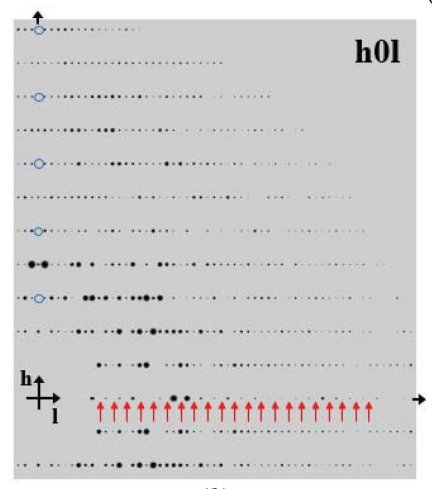

(b)

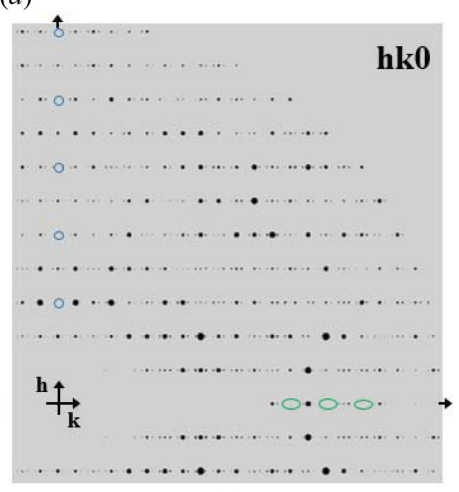

(c)
Figure 4

Pseudo-precession photographs of diffraction data from the gMod3 crystal (see Table 1). Diffraction data, main reflections and satellites, were integrated using Eval15 as described previously (Porta et al., 2011). In part (a) the $0 k l$ plane is displayed with portions of the $k$ axis, $l$ axis and centre portion magnified. Systematic absences along $k$ are highlighted with green ovals and along $l$ with red circles. In the centre portion zoom, red rectangles highlight reflections where the satellites do not have equal intensity, green rectangles show where there is just a main reflection with no satellites, and blue rectangles show examples where the satellites extinguish the main reflection. Part $(b)$ shows the $h 0 l$ plane and part $(c)$ the $h k 0$ plane, with systematic absences along $h$ circled in blue, along $l$ highlighted with red arrows and along $k$ with green ovals. To prepare these pseudo-precession photographs, the reflections were reindexed to a supercell using an Awk script to reindex the $k$ reflections under the supercell condition $k=7 k+2 m$, where $m$ is the satellite order ( $m= \pm 1$ in this study). Reindexing the data into a supercell is illustrated in Fig. S1 of the supporting information. The reindexed reflections were converted to realistic pseudo-precession photographs with the MLFSOM software, which applies a point-spread function (Holton, 2008; Holton et al., 2012). 
Table 2

Merging $R$ values between datasets.

Calculated with main reflections only.

\begin{tabular}{lllll}
\hline & Periodic & xMod1 & xMod2 & gMod3 \\
\hline Periodic & 0 & 0.371 & 0.616 & 0.307 \\
xMod1 & & 0 & 0.665 & 0.284 \\
xMod2 & & & 0 & 0.738 \\
gMod3 & & & & 0
\end{tabular}

Calculated with main reflection intensities added to satellites.

\begin{tabular}{lllll}
\hline & Periodic & xMod1 & xMod2 & gMod3 \\
\hline Periodic & 0 & 0.362 & 0.372 & 0.292 \\
xMod1 & & 0 & 0.281 & 0.281 \\
xMod2 & & 0 & 0.255 \\
gMod3 & & & & 0 \\
\hline
\end{tabular}

$R_{\text {merge }}=\sum_{h k l}|| F_{\text {periodic }}|-| F_{\text {modulated }}|| / \sum_{h k l}\left|F_{\text {periodic }}\right| . R_{\text {merge }}<0.1$ means the datasets are very isomorphous. If $R_{\text {merge }}>0.3$ there is a lack of isomorphism between the crystals. The periodic crystallographic data were reported by Porta \& Borgstahl (2012) as the 'wideopen-state' crystal.

reflections the $R_{\text {merge }}$ values improve and fall in a range of $25-$ $37 \%$, still not isomorphous. Clearly the three modulated structures are significantly different from each other and from the periodic crystal. The intensity of the modulation is also indicated by the strength of the satellite intensities (e.g. in Table $1 I / \sigma$ for the satellite reflections ranks their strength as follows: $\mathrm{gMod} 3>\mathrm{xMod} 2=\mathrm{xMod} 1)$.

We have three cryocooled incommensurately modulated PA structures to solve of varying modulation strength (Fig. 3). When we look at the gMod3 crystal more closely in pseudoprecession photographs, it can be seen how the satellites relate to the main reflections (Fig. 4). Satellites are not always present (green rectangles, Fig. $4 a$ ), do not have to be of equal intensity (red rectangles, Fig. $4 a$ ) and can extinguish the main reflections (blue rectangles, Fig. 4a). The relative intensities between the satellite and the main reflections are analysed by resolution bin in Table 3 for the gMod3 crystal (see also Fig. 7 and Table 2 of Porta et al., 2011). The ratio of the satellite to main reflection intensity is lower in low-resolution bins and increases in the high-resolution bins. This is a general feature of modulated PA crystals.
Table 3

Statistics on the relative intensity of satellite-to-main reflections for crystal gMod3.

\begin{tabular}{llllll}
\hline $\begin{array}{l}\text { Resolution } \\
\text { bin }(\AA)\end{array}$ & $\begin{array}{l}\text { No. of } \\
\text { reflections }\end{array}$ & $\begin{array}{l}\text { Average } \\
\text { ratio } \dagger\end{array}$ & $\begin{array}{l}\text { Standard } \\
\text { deviation ratio }\end{array}$ & $\begin{array}{l}\text { Maximum } \\
\text { ratio }\end{array}$ & $\begin{array}{l}\text { Rejects } \\
\text { (ratio > 1) }\end{array}$ \\
\hline $26.88-5.66$ & 8931 & 0.14 & 0.19 & 25.53 & 715 \\
$5.66-4.45$ & 8180 & 0.22 & 0.24 & 27.45 & 1467 \\
$4.45-3.87$ & 7645 & 0.26 & 0.25 & 42.01 & 2001 \\
$3.87-3.49$ & 6941 & 0.31 & 0.27 & 21.77 & 2705 \\
$3.49-3.21$ & 6753 & 0.31 & 0.27 & 12.58 & 2893 \\
$3.21-3.00$ & 6686 & 0.33 & 0.28 & 13.28 & 2961 \\
$3.00-2.82$ & 6874 & 0.35 & 0.28 & 8.20 & 2772 \\
$2.82-2.67$ & 6925 & 0.38 & 0.29 & 19.74 & 2721 \\
$2.67-2.52$ & 7221 & 0.39 & 0.29 & 6.31 & 2426 \\
$2.52-2.36$ & 7443 & 0.41 & 0.28 & 6.02 & 2202 \\
\hline
\end{tabular}

$\dagger$ Ratio $=I_{\text {satellite }} / I_{\text {main }}$. Only reflections with strong main reflections $\left[I_{\text {main }} / \sigma\left(I_{\text {main }}\right)>2.5\right]$ were used. The average and standard deviations were calculated from the ratio distribution after reflections with a ratio greater than 1 had been rejected. $\ddagger$ Cases where $I_{\text {satellite }}>I_{\text {main }}$ were excluded from the calculation. A similar table for the xMod1 crystal was published by Porta et al. (2011).

\section{Assignment of a superspace group to a protein crystal}

A general procedure for the assignment of a superspace group is given by Janssen et al. (1999). These steps are analysed here with our PA diffraction data and the description of the process is streamlined to include only the symmetry elements found in chiral molecule crystals. Hopefully this example will make these methods more accessible to protein crystallographers.

\subsection{Determine the Laue class and crystallographic point group}

The Laue group of the diffraction pattern is the point group in three dimensions that transforms every diffraction peak into a peak of the same intensity (except for deviations from Friedel's law caused by dispersion) (Rupp, 2010). For biological crystals there are 11 Laue symmetry classes and 11 chiral crystallographic point groups (32 point groups for small molecular crystals). These are triclinic 1 , monoclinic 2 , orthorhombic 222 , tetragonal 4 or 422 , trigonal 3 or 32 , hexagonal 6 or 622, and cubic 23 or 432 (Table S1 in the supporting information). Processing of the main diffraction data with $D^{*} T R E K$ (Table 4) or with Eval15 (Table 5) shows that the Laue class is $\mathrm{mmm}$ and the point group is 222

Table 4

Processing of main reflections with $d^{*} T R E K$ for crystal xMod1.

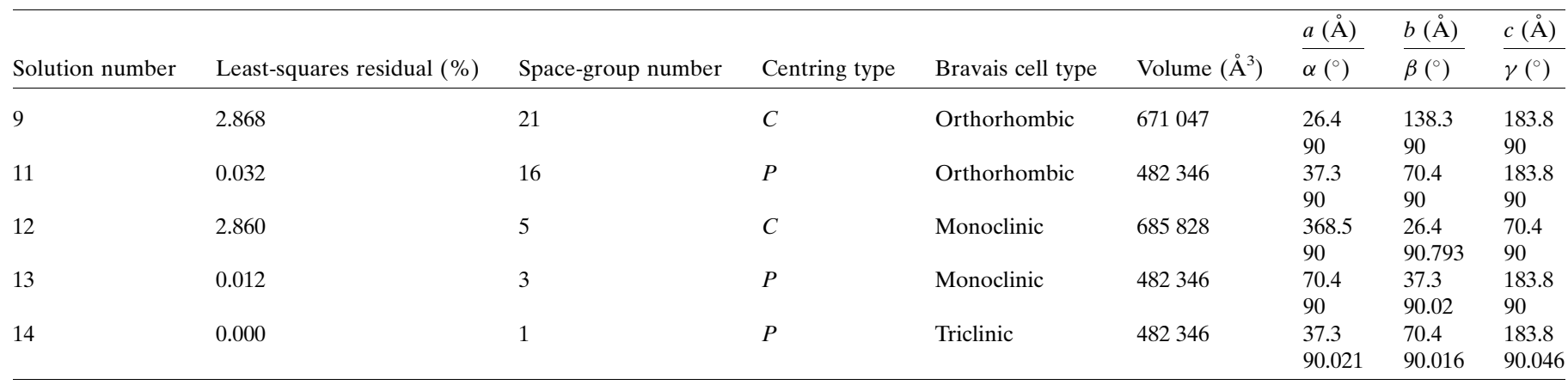


Table 5

Data processing with Eval15 for crystal xMod1.

\begin{tabular}{llllrrrl}
\hline Point group & $R$ & $R_{\text {meas }}$ & $R_{\text {pim }}$ & \multicolumn{1}{c}{$\chi^{2}$} & $n$ Uni & $n R_{\text {sym }}$ & Bravais cell type \\
\hline$\overline{1}$ & 0.134 & 0.183 & 0.125 & 7.95 & 23912 & 53619 & Triclinic \\
$2 / m$ & 0.141 & 0.186 & 0.118 & 9.66 & 25996 & 71467 & Monoclinic \\
$m m m$ & 0.119 & 0.153 & 0.095 & 10.81 & 28097 & 87773 & Orthorhombic \\
$4 / m$ & 1.124 & 1.229 & 0.484 & 1233.4 & 17037 & 85198 & Tetragonal low \\
$4 / m m m$ & 1.207 & 1.293 & 0.453 & 1198.5 & 12459 & 88763 & Tetragonal high \\
$\overline{3}$ & 0.838 & 0.991 & 0.510 & 790.3 & 22881 & 75520 & Trigonal low \\
$\overline{3} m 1$ & 1.117 & 1.249 & 0.537 & 1155.4 & 18061 & 86616 & Trigonal high \\
$\overline{3} 1 m$ & 1.136 & 1.255 & 0.511 & 1138.3 & 16767 & 86629 & Trigonal high \\
$6 / m$ & 0.800 & 0.887 & 0.368 & 701.4 & 19586 & 86619 & Hexagonal low \\
$6 / m m m$ & 1.183 & 1.255 & 0.404 & 1051.7 & 11045 & 88313 & Hexagonal high \\
$m 3$ & 1.356 & 1.477 & 0.568 & 1144.9 & 14924 & 88245 & Cubic low \\
$m 3 m$ & 1.338 & 1.390 & 0.346 & 757.8 & 14819 & 88916 & Cubic high \\
\hline
\end{tabular}

$R=\sum\left(\left|I_{i}-I_{\text {mean }}\right|\right) / \sum\left(I_{i}\right), \quad R_{\text {meas }}=[n /(n-1)]^{1 / 2} \sum\left(\left|I_{i}-I_{\text {mean }}\right|\right) / \sum\left(I_{i}\right), \quad R_{\text {pim }}=$ $[1 /(n-1)]^{1 / 2} \sum\left(\left|I_{i}-I_{\text {mean }}\right|\right) / \sum\left(I_{i}\right), \chi^{2}=(1 / n) \sum\left[\left(\left|I_{i}-I_{\text {mean }}\right|\right) / \sigma_{i}\right]^{2}, n$ Uni is the number of unique reflections and $n R_{\mathrm{sym}}$ is the number used to calculate the $\mathrm{R}$ values.

(Pflugrath, 1999; Schreurs et al., 2010). There are only 23 $(3+1) \mathrm{D}$ superspace groups with this symmetry.

\subsection{Find the basic unit cell for the main reflections and a modulation wavevector}

The main reflections are separated from the satellites, usually by intensity, and indexed. Reflection extinctions are used to select the Bravais class for the main reflections (Fig. S2). Note that only noncubic classes are possible for $(3+1) \mathrm{D}$ modulations because a one-dimensional incommensurate modulation is incompatible with cubic symmetry. The satellites are usually assigned to the main reflections (can be extinct) that they are closest to. Then the direction and dimensions of the $\mathbf{q}$ vector are determined by fitting the satellites. If possible, it is preferable to place the $\mathbf{q}$ vector along a reciprocal lattice vector.

PA crystals are of the primitive orthorhombic Bravais lattice. This can be seen in the analysis of just the main reflections (Table 4 , solution 11). Centring-type $P$ orthorhombic has a low least-squares residual almost as low as $P$ triclinic or $P$ monoclinic. $C$ centring is ruled out by the large least-squares residual. Eval15 processing also selects primitive orthorhombic as the Bravais lattice (Table 5). This narrows the assignment down to 15 superspace groups. Eval15 was used to define the $\mathbf{q}$ vector, which is in the direction of $\mathbf{b}^{*}$, for each crystal (Porta et al., 2011). We note that the magnitude of the $\mathbf{q}$ vector for $\mathrm{xMod} 1$ is close to $2 / 7=0.2857 \ldots$ and so $2 / 7$ was used as an approximation when the diffraction was reindexed for display as a pseudo-precession in Fig. 4 (see also Fig. S1).

\subsection{Determine the 3D space group of the average structure}

The average structure is commonly found by using the main reflections only and corresponds to averaging the contents of several unit cells in three dimensions. The space group of the average structure is determined from the main reflections. This helps make a good choice for the starting structure in superspace refinement. Tables 4 and 5 show that the three-dimensional space group is $P 222$. The three-dimensional space group for the average structure is determined from the main reflections. In our case, checks for centring rule out $C, F$ or $I$ and the lattice is primitive. Extinctions along $h, k$ and $l$ (Fig. 4) indicate the presence of screw axes along all three dimensions. Since the data are of fairly low resolution the assignment of the space group was checked by performing molecular replacement with just the main reflections using MOLREP (Table 6) (Vagin \& Teplyakov, 2000). This settles any uncertainty and the $3 \mathrm{D}$ space group of the average structure is $P 2_{1} 2_{1} 2_{1}$.

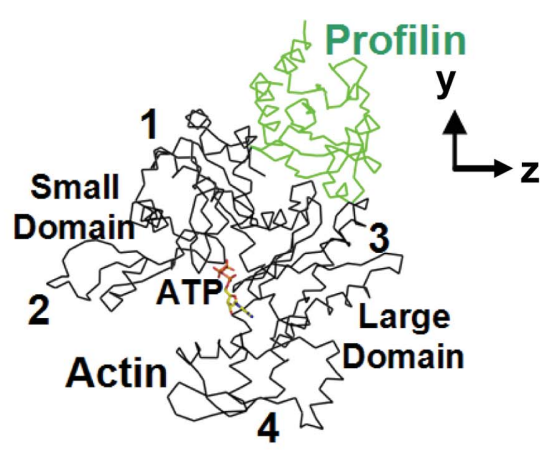

(a)

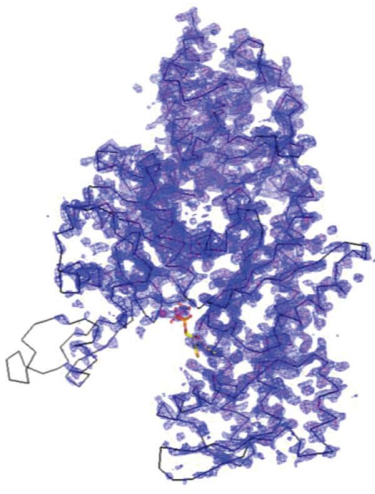

(b)

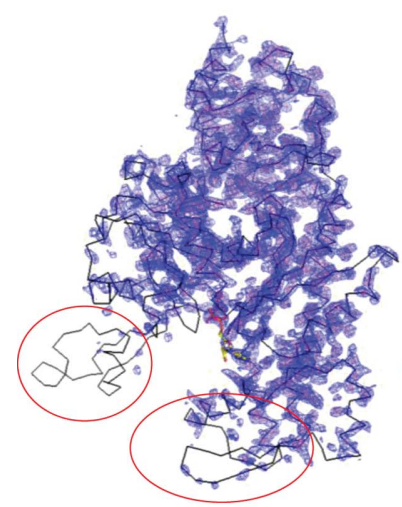

(d)

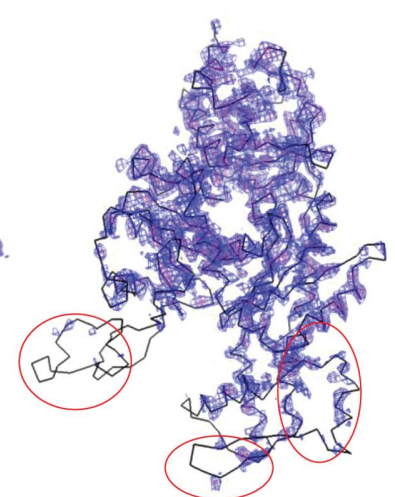

(c)

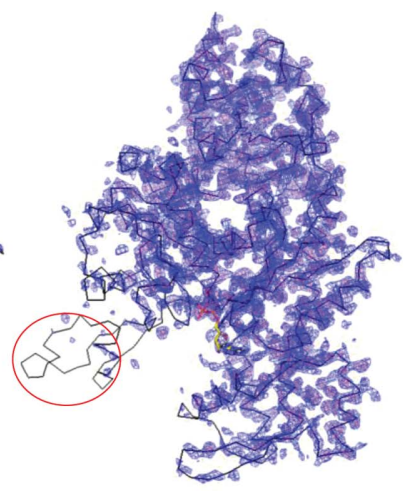

(e)
Figure 5

Analysis of the average structure in modulated PA crystals. (a) $\mathrm{C} \alpha$ trace of profilin:actin with the domains of actin labelled. (b)-(e) $2 F_{\mathrm{o}}-F_{\mathrm{c}}$ electron density $(2 \sigma$, blue; $3 \sigma$, red) of $(b)$ wide-open state, periodic structure at $2.3 \AA$ resolution, and $(c) \mathrm{xMod} 1,(d) \mathrm{xMod} 2$ and $(e) \operatorname{gMod} 3$ average modulated structures (main reflections + satellites merged with $J A N A 2006)$. Regions in the average maps with density that is weaker than in the periodic map (circled in red) are the locations in the structure with multiple positions owing to modulation. 
At this point a refinement of the average structure can be performed and the resulting electron density observed. The average structures refined with $R E F M A C$ crystallographic $R$ values of $27-28 \%$ (Murshudov et al., 1997). The electron density of the average structure (Fig. 5) reveals that some parts of the structure are modulated more than others. The average electron density for profilin and subdomains 1 and 3 of actin are fairly well ordered. Actin domains 2 and 4 have very weak density, and this indicates that their motions are more dramatic in the modulation wave. The modulation function in actin appears to be more pronounced than that in profilin.

An illustration of the crystal contacts in PA crystals shows how the modulated regions correspond to the crystal directions (Fig. 6). For the PA case, the indexing showed that the modulation is along $\mathbf{b}$ (collinear with $\mathbf{y}$ ) in the crystal, which corresponds to an 'actin ribbon' formed by the crystal lattice (Schutt et al., 1991). It is likely that the protein undergoes a conformational change that affects the neighbouring PA molecules in such a way as to produce the observed modulation in the diffraction pattern (Schutt et al., 1991). The struc-

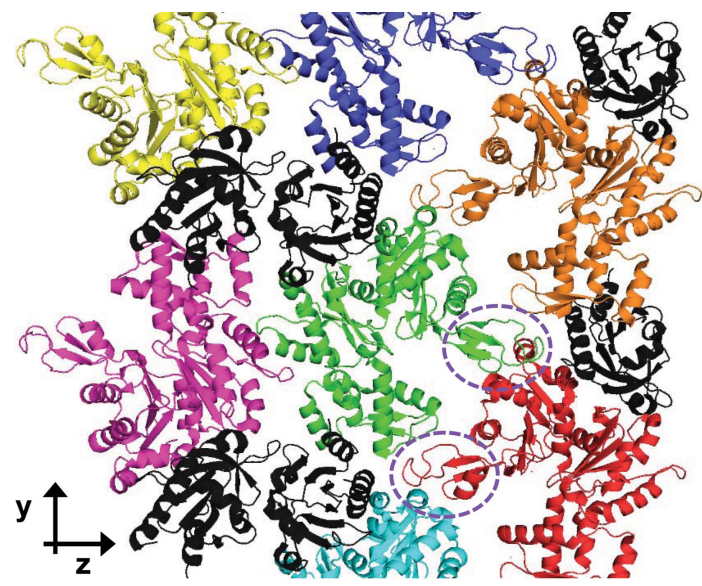

(a)

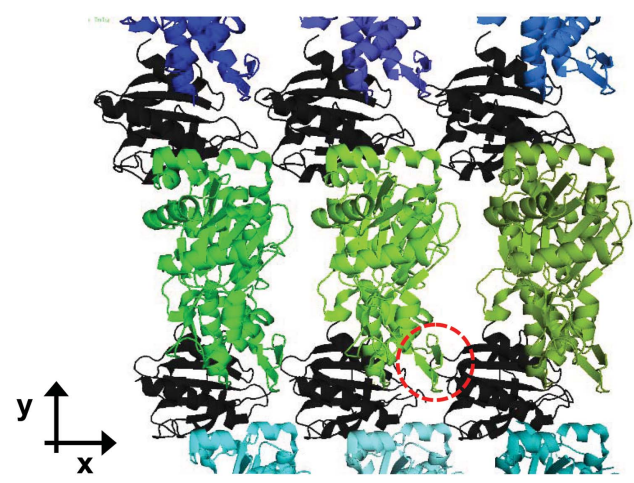

(b)

Figure 6

The crystal lattice of profilin:actin crystals (a) looking down $\mathbf{x}$ and $(b)$ looking down $\mathbf{z}$. In both views the direction of the actin ribbon formed in these crystals is along $\mathbf{y}$ and vertical. In (a) one complete actin ribbon is seen on the right half of the figure, composed of four profilins (black) and five actins (cyan, red, green, orange and blue). Domain 2 in the actin small domain is indicated with a dashed circle in magenta in part $(a)$ and red in part $(b)$.
Table 6

Check of space-group screw axes with Molrep with main reflections for crystal xMod1.

The score is the product of the correlation coefficient (CC) and packing function $[Q(s)]$.† The contrast is the ratio of the top score to the mean score.

\begin{tabular}{llll}
\hline Space group & Space-group number & Score $^{\ddagger}$ & Contrast \\
\hline$P 222$ & 16 & 0.101 & 0 \\
$P 222_{1}$ & 17 & 0.407 & 6.899 \\
$P 2_{1} 22$ & 1017 & 0.100 & 0 \\
$P 22_{1} 2$ & 2017 & 0.086 & 0 \\
$P 2_{1} 2_{1} 2$ & 18 & 0.107 & 0 \\
$P 2_{1} 22_{1}$ & 2018 & 0.430 & 9.494 \\
$P 22_{1} 2_{1}$ & 3018 & 0.437 & 11.505 \\
$P 2_{1} 2_{1} 2_{1}$ & 19 & 0.632 & 18.925 \\
\hline
\end{tabular}

$\dagger$ Score $=Q(s) \times \mathrm{CC}$, where $Q(s)=-\sum_{j \neq k} \int \rho_{j}(r, s) \rho_{k}(r, s) \mathrm{d} r$ and $\mathrm{CC}=\left\langle\left|F_{\mathrm{o}}\right|\left|F_{\mathrm{c}}\right|-\right.$ $\left.\left\langle\left|F_{\mathrm{o}}\right|\right\rangle\left\langle\left|F_{\mathrm{c}}\right|\right\rangle\right\rangle /\left(\left\langle\left|F_{\mathrm{o}}\right|^{2}-\left\langle\left|F_{\mathrm{o}}\right|\right\rangle^{2}\right\rangle\left\langle\left|F_{\mathrm{c}}\right|^{2}-\left\langle\left|F_{\mathrm{c}}\right|\right\rangle^{2}\right\rangle\right)^{1 / 2}$.

tural basis for the modulated PA diffraction pattern has not yet been determined.

\subsection{Identification of the $(3+1) \mathrm{D}$ Bravais lattice type}

The $(3+1) \mathrm{D}$ Bravais class is determined by the 3D Bravais class and the components $\alpha, \beta, \gamma$ of $\mathbf{q}$. Next we find the superspace group compatible with the previously derived results and with the special extinctions observed in the diffraction pattern. In Table S2 there are 15 orthorhombic $(3+1)$ D superspace groups (Nos. 16.1-19.1). From the main reflections we know our lattice is primitive and there is no centring. There are three screw axes. Applying the screw axes narrows the selection down to one and the superspace group for the incommensurately modulated PA crystals is number 19.1 with notation $P 2_{1} 2_{1} 2_{1}(00 \gamma)$. There are actually three related versions of this space group: $P 2_{1} 2_{1} 2_{1}(\alpha 00)$, $P 2_{1} 2_{1} 2_{1}(0 \beta 0)$ and $P 2{ }_{1} 2_{1} 2_{1}(00 \gamma)$. The direction of the modulation is shown by the position of the coefficients. For PA the orientation is $P 2{ }_{1} 2_{1} 2_{1}(0 \beta 0)$. The symmetry operators need to be modified to work with modulation along this axis relative to

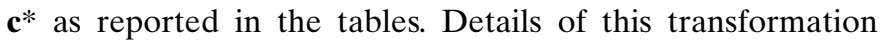
were reported by Lovelace et al. (2013).

\section{Conclusions}

After integration of reflections in Eval15 the unit-cell dimensions and $\mathbf{q}$ vector length and direction are known. The final test of the superspace group assignment comes in the next step when it is applied to the integrated diffraction data via the $S A D A B S$ software (Sheldrick, 1996). The program workflow is presented in Fig. 3 of Porta et al. (2011). It can be seen in Table 1 that the $R_{\text {sym }}$ values obtained from $S A D A B S$ look reasonable and are quite good for the well measured data of $<4 \AA$ resolution.

During data collection it was noticed that particularly strong satellite reflections were associated with extinguished main reflections (Fig. 4). As it turns out, this is indicative of large movements in the structural modulation (Janssen et al., 1999). It is interesting to note that, when normal periodic actin 
in PA crystals undergoes a transition from the 'open' to 'tight' state, the unit-cell dimension $c$ changes by $14 \AA$, yet the crystals are stable (Chik, 1996). It is therefore possible that the structural transitions needed to bring about such a large modulation might be on a similar scale, especially those involving actin subdomains 1 and 4 . Refinement of the incommensurate PA structures will inevitably shed light on the nature of these higher-order actin structures and provide insight into the early stages of actin filament formation. This is the next step in our research and involves further software development for crystallographic refinement of a protein in a $(3+1) \mathrm{D}$ superspace group.

\section{Acknowledgements}

We would like to thank Clarence Schutt at Princeton University and Uno Lindberg at Stockholm University for introducing us to PA crystals and many useful discussions. We thank Dr Sander Van Smaalen at the Universität Bayreuth, and Dr Václav Petříček, Dr Michal Dušek and Dr Lukáš Palatinus at the Academy of Sciences of the Czech Republic, Prague, for being patient teachers. We thank Dr James Holton at Lawrence Berkley National Laboratory for help in making the pseudo-precession figures and Kelly Jordan for help in constructing tables.

\section{Funding information}

Funding for this research was provided by: National Science Foundation, Division of Molecular and Cellular Biosciences (award Nos. MCB-0718661, MCB-1518145, CNIC-IIA1404976); Nebraska Research Initiative.

\section{References}

Carlsson, L. (1979). PhD thesis, Uppsala University, Sweden. Chik, J. K. (1996). PhD thesis, Princeton University, NJ, USA. Chik, J. K., Lindberg, U. \& Schutt, C. E. (1996). J. Mol. Biol. 263, 607623.

Dauter, Z. \& Jaskolski, M. (2010). J. Appl. Cryst. 43, 1150-1171. Helliwell, J. R. (2008). Crystallogr. Rev. 14, 189-250.
Holton, J. M. (2008). Acta Cryst. A64, C77.

Holton, J. M., Nielsen, C. \& Frankel, K. A. (2012). J. Synchrotron Rad. 19, 1006-1011.

Janssen, T., Janner, A., Looijenga-Vos, A. \& Wolff, P. M. D. (1999). International Tables for Crystallography, Vol. C, Mathematical, Physical and Chemical Tables, edited by A. J. C. Wilson \& E. Prince, pp. 899-947. Dordrecht: Kluwer Academic Publishers.

Lovelace, J. J., Murphy, C. R., Daniels, L., Narayan, K., Schutt, C. E., Lindberg, U., Svensson, C. \& Borgstahl, G. E. O. (2008). J. Appl. Cryst. 41, 600-605.

Lovelace, J. J., Simone, P. D., Petrícek, V. \& Borgstahl, G. E. O. (2013). Acta Cryst. D69, 1062-1072.

Lovelace, J. J., Winn, M. D. \& Borgstahl, G. E. O. (2010). J. Appl. Cryst. 43, 285-292.

Murshudov, G. N., Vagin, A. A. \& Dodson, E. J. (1997). Acta Cryst. D53, 240-255.

Oda, T., Makino, K., Yamashita, I., Namba, K. \& Maéda, Y. (2001). Biophys. J. 80, 841-851.

Orlov, I., Palatinus, L. \& Chapuis, G. (2008). J. Appl. Cryst. 41, 11821186.

Pflugrath, J. W. (1999). Acta Cryst. D55, 1718-1725.

Porta, J. (2011). PhD thesis, University of Nebraska Medical Center, Omaha, NE, USA.

Porta, J. C. \& Borgstahl, G. E. (2012). J. Mol. Biol. 418, 103-116.

Porta, J., Lovelace, J. J., Schreurs, A. M. M., Kroon-Batenburg, L. M. J. \& Borgstahl, G. E. O. (2011). Acta Cryst. D67, 628-638.

Rupp, B. (2010). Biomolecular Crystallography: Principles, Practice, and Application to Structural Biology. New York: Garland Science/ Taylor and Francis Group.

Schreurs, A. M. M., Xian, X. \& Kroon-Batenburg, L. M. J. (2010). J. Appl. Cryst. 43, 70-82.

Schutt, C., Lindberg, U. \& Myslik, J. (1991). Nature, 353, 508.

Schutt, C. E., Lindberg, U., Myslik, J. \& Strauss, N. (1989). J. Mol. Biol. 209, 735-746.

Schutt, C. E., Myslik, J. C., Rozycki, M. D., Goonesekere, N. C. \& Lindberg, U. (1993). Nature, 365, 810-816.

Sheldrick, G. M. (1996). SADABS. University of Göttingen, Germany.

Smaalen, S. van (2005). Z. Kristallogr. 219, 681-691.

Smaalen, S. van (2007). Incommensurate Crystallography. Oxford University Press.

Smaalen, S. van, Campbell, B. J. \& Stokes, H. T. (2013). Acta Cryst. A69, 75-90.

Stokes, H. T., Campbell, B. J. \& van Smaalen, S. (2011). Acta Cryst. A67, 45-55.

Vagin, A. \& Teplyakov, A. (2000). Acta Cryst. D56, 1622-1624.

Wagner, T. \& Schönleber, A. (2009). Acta Cryst. B65, 249-268. 